\title{
Physically Motivated Enhancement of Color Images for Fiber Endoscopy
}

\author{
Christian Winter ${ }^{1}$, Thorsten Zerfaß ${ }^{2}$, Matthias Elter ${ }^{2}$, Stephan Rupp ${ }^{2}$, \\ and Thomas Wittenberg ${ }^{2}$ \\ ${ }^{1}$ University Erlangen-Nuremberg, Chair for Information Technology, \\ Am Wolfsmantel 33, Erlangen, Germany \\ wnt@like.e-technik. uni-erlangen.de \\ ${ }^{2}$ Fraunhofer-Institute for Integrated Circuits IIS, \\ Am Wolfsmantel 33, Erlangen, Germany \\ $\{$ zfs, elt,rupp, wbg\}@iis.fraunhofer.de
}

\begin{abstract}
Fiber optics are widely used in flexible endoscopes which are indispensable for many applications in diagnosis and therapy. Computeraided use of fiberscopes requires a digital sensor mounted at the proximal end. Most commercially available cameras for endoscopy provide the images by means of a regular grid of color filters what is known as the Bayer Pattern. Hence, the images suffer from false colored spatial moiré, which is further stressed by the downgrading fiber optic transmission yielding a honey comb pattern. To solve this problem we propose a new approach that extends the interpolation between known intensities of registered fibers to multi channel color applications. The inventive idea takes into account both the Gaussian intensity distribution of each fiber and the physical color distribution of the Bayer pattern. Individual color factors for interpolation of each fiber area make it possible to simultaneously remove both the comb structure from the fiber bundle as well as the Bayer pattern mosaicking from the sensor while preserving depicted structures and textures in the scene.
\end{abstract}

\section{Introduction}

Flexible endoscopy based on thin fiberscopes is widely used for medical diagnosis and therapy. To digitize and process the image data the eye-piece is connected to a video camera. For visualization and further computer aided applications, e.g. image enhancement, computer-aided diagnosis (CAD) or navigation through natural orifices, a preferably accurate and true color representation of the scene is desired. Particularly with regard to detection and classification of lesions, texture and color are important components for the correct decision.

Commonly used single-chip cameras compose color images by means of a photo sensitive sensor array with Bayer pattern. On these regular grids (cf. fig. 1(d)], each pixel element is masked by a specific color filter. If a colored part of an image is not equally distributed to these three basic colors, it cannot be accurately

\footnotetext{
${ }^{1}$ The basic color components are red, green and blue, where green is represented twice, since the human eye is more sensitive to the according wavelength.
} 
demosaicing. This kind of moiré becomes particularly worse in combination with color imaging through fiber bundles. The image bundle of a fiberscope contains a structured set of small fibers, usually between 5,000 to 20,000, depending on the working diameter of the fiberscope. Due to the hexagonal distribution of the fibers in the bundle and the Cartesian distribution of the sensor's elements, the dimension of the digital sensor typically exceeds the number of fibers by a factor of 10 to 100 to avoid spatial moiré. This results in the typical comb structure (see fig. 1(a) . If one fiber would be directly mounted to one sensor element, its intensity could be determined exactly, but due to the color pattern it only works for one color component. To obtain the color value we need several different sensor elements to be illuminated. The drawback hereof will be an unbalancing of color elements and therefore false colors will be unavoidable (see fig. 1(a-c)). Usually the contribution to each color is statistically distributed, but fiber transmission suffers from irregular occlusion through cladding and micro-shading.

This work introduces a new approach taking into account both, the knowledge about the illumination center of every single fiber in the image on the sensor as well as the underlying color coding by the Bayer pattern. By processing the raw image data of the sensor it is possible to simultaneously remove both the comb structure from the fiber bundle and the Bayer pattern mosaicking from the sensor while preserving depicted structures and textures in the scene.

Sect. 2 presents the state of the art of relevant imaging and image processing. The proposed method of physically motivated enhancement of color images

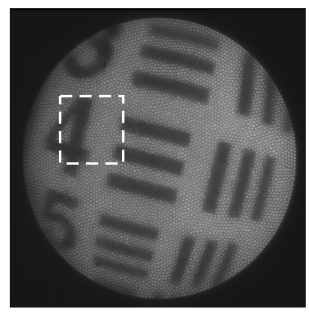

(a)

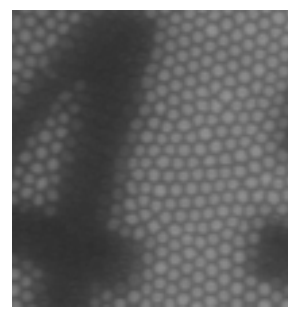

(b)

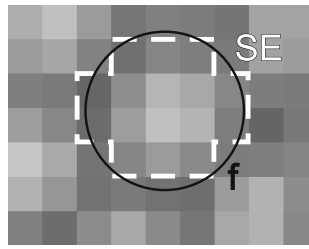

(c)

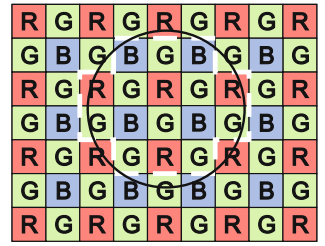

(d)

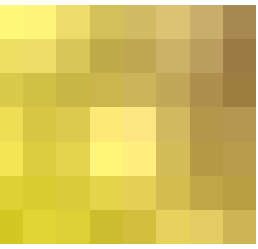

(e)

Fig. 1. Reference chart captured by quartz glass fiberscope (a) with enlarged section (b). Enlarged adjacencies of a single fiber $f$ with assigned sensor elements SE (c). Schematic section of color pattern on CCD video sensor with denoted fiber boundary (d). Resulting color distribution after sampling section c by Bayer pattern d (c). 
for fiber endoscopy is described in Sect. 3. Experiments and results in Sect. 4 show the progress in visual quality of the approach for a typical example. The conclusions in Sect. 5 summarize the work and discuss future research.

\section{Related Work}

Literature covers several techniques to enhance the visual quality of fiber optic imaging. Algorithms which dispose of the comb structure can be categorized into two main classes with respect to the working domain.

In the spatial domain there are attempts to recover the image by nonlinear diffusion 1, edge preserving smoothing and various algorithms with the idea of interpolation 23]. The subpixel accurate registration of fibers with respect to the physical motivation of the intensity distribution in their cross section can be used to apply super resolution approaches on non-uniform distributed grids [4,5].

In the frequency and wavelet domain, characterizable structures are reduced by dedicated bandpass filtering. [1] gives an overview of useful basic methods to remove typical structures from fiberscopic images. Manually parametrized [6] and also automatically adapted masks [7] can be used for fiber processing. The latter work suggests star shaped masks for optimal results with coherent glass fiber bundles. We consider this method as reference algorithm for filtering to evaluate our method.

The importance of color [8] and several ideas of its calibration for endoscopy are pointed out in literature as well as comprehensive work about demosaicing of video data from sensors with Bayer pattern. However, no approach can been found which takes into account the information about the Bayer pattern on the image sensor to add knowledge about the formation and exact distribution of colors in each fiber's image. Our work particularly utilizes this physical background to enhance color interpolation for fiber optic imaging. Other critical aspects of fiber imaging in endoscopy, like distortion and luminance correction are addressed in various articles but are not of special interest for this work.

\section{Method}

The physically motivated enhancement of color images in fiber endoscopy is performed in four steps, where the first two are for initialization. First all fibers are localized with subpixel accuracy on the sensor grid (Sec. 3.1). This information is then used to determine factors for local color correction (Sec. 3.2). Step three performs the false color removal (Sec. 3.3), while the last step interpolates on adjusted color values (Sec. [3.4). The result is a comb-free, true color image of the scene.

\subsection{Fiber Registration}

The method described in [3] is applied to determine each fiber's center. Subpixel accuracy is obtained by fitting a Gaussian function to the intensity distribution 
of the linear interpolated green channel of an empty bright image, which is referred to as the white image. From a mathematical and physical point of view it is important to control the camera's gain and shutter to just not saturate the image's peaks 2 . All fibers that are not saturated and fulfill the criteria of mean distance to the nearest neighbors (see 3 for details) are taken as valid and stored in a Delaunay grid to serve the following steps.

\subsection{Color Distribution and Correction Factors}

Determination of the local color distribution and calculation of according correction factors is done by the following procedure:

1. Assignment to closest fiber: With the information of fiber registration all sensor elements SE now can be related to their nearest fiber (see fig. 1(c)]. From the amount $F$ of all fibers, for the fiber $f$ in the white image these are the elements $\operatorname{SE}(f)$ with $f \in F$.

2. Accumulation of sensor intensities: For each channel $i$, the intensities $I$ of all related sensor elements are accumulated to the calibration sum $K$.

$$
K_{i}(f)=\sum_{S E(f)} I_{i}(f) \quad \text { with } \quad i \in\{R, G, B\} \quad \text { and } \quad f \in F
$$

3. Determination of reference illumination: A reference illumination $\hat{K}(f)$ is defined to compensate global intensity gradients and enhance the visual perception. Here we use a constant value, but in a more sophisticated version it is adapted to local information.

4. Calculation of correction factors: The intensity sums are referred to the reference level and result in the correction factor

$$
C_{i}(f)=\frac{\hat{K}(f)}{K_{i}(f)} \quad \text { with } \quad i \in\{R, G, B\} \quad \text { and } \quad f \in F
$$

\subsection{False Color Reversal}

The last two steps are performed for a whole sequence of images either from a live stream, an image archive or other image stacks. Without loss of generality they are described for a single image frame.

1. Intensity sum per channel: Similar to the accumulation of sensor intensities during the initialization, here for all related sensor elements $\mathrm{SE}(f)$ of one fiber $f$ the intensities $I$ from within the current image are accumulated to the sum $S$ :

$$
S_{i}(f)=\sum_{S E(f)} I_{i}(f) \quad \text { with } \quad i \in\{R, G, B\} \quad \text { and } \quad f \in F .
$$

${ }^{2}$ Meaning that the maximum pixel intensity is equal or lower than the sensor's capacity. 
2. Real fibers' intensity per channel: The corrected fiber intensity $\hat{I}$ for the channel $i$ of the fiber $f$ in the current image is given by the product of the sum $S_{i}(f)$ and the correction factor $C_{i}(f)$.

$$
\hat{I}_{i}(f)=S_{i}(f) \cdot C_{i}(f) \quad \text { with } \quad i \in\{R, G, B\} \quad \text { and } \quad f \in F
$$

3. Fiber related color value: The fiber $f$ is now represented by the intensity triple $I_{C}(f)=\left[\hat{I}_{R}(f), \hat{I}_{G}(f), \hat{I}_{B}(f)\right] \quad$ for $\quad f \in F$

\subsection{Color Interpolation}

With the corrected color value of Sect. 3.3, the intensity based barycentric interpolation for fiberscopic data 3 is extended to color processing. The utilized Delaunay grid is supplemented with additional color information for each fiber in terms of the adjusted intensity triple $I_{C}^{k, f}$.

Performing the image processing steps on the highest possible quantization within the technical specs of the camera sensor (e.g. 12 or 14 bit) and preserving the digital precision of intensity data until the final barycentric weighting ensures the least roundoff error in the result. To ensure real time performance we precalculate a lookup table (LUT) for fast access to fiber and color information for each output image pixel. Pixels which are not in between three valid fiber centers are skipped for processing the LUT.

\section{Experimental Results}

To exemplify the impact of the proposed method we use the example of human skin (fig. 2(A1)). Although particular details of the algorithm can be better understood by using calibration targets or artificial textures, we go for this surface as a good trade-off to show the handling with color and relief structure at the same time on texture tissue. We use a standard flexible endoscope with glass fiber image transmission (Schölly Fiberoptic GmbH). The working diameter is $3.8 \mathrm{~mm}$ and the direct sight offers a field of view of $80^{\circ}$. The working distance is between $5 \mathrm{~mm}$ and $20 \mathrm{~mm}$. The technical specs specify the number of fibers in the optic bundle with 10,000 .

A typical fiber registration as described in Sect. 3.1 locates about $95 \%$ of all fibers. This percentage is reasonable since from a technical point of view not all fibers are used for imaging and from a signal processing point of view some fibers are not suitable for registration since the distribution is not representative or separable. $99.96 \%$ of the detected fibers are taken as valid with respect to the criteria stated in 3.1. The mean distance between all valid fibers is $\mu=5.20 \mathrm{px}$ (pixel) with a variance of $\sigma=0.652 \mathrm{px}$. The effect of false colors depends on the relation between sensor resolution and the number of fibers. We use a typical scenario here but made similar experiences with various settings of cameras and different endoscopes.

Figure 2(A1) shows the color image (with 8 bit color depth for each of the three channels) recorded by a digital video camera with dimension of $800 \times 600 \mathrm{px}$. 
1
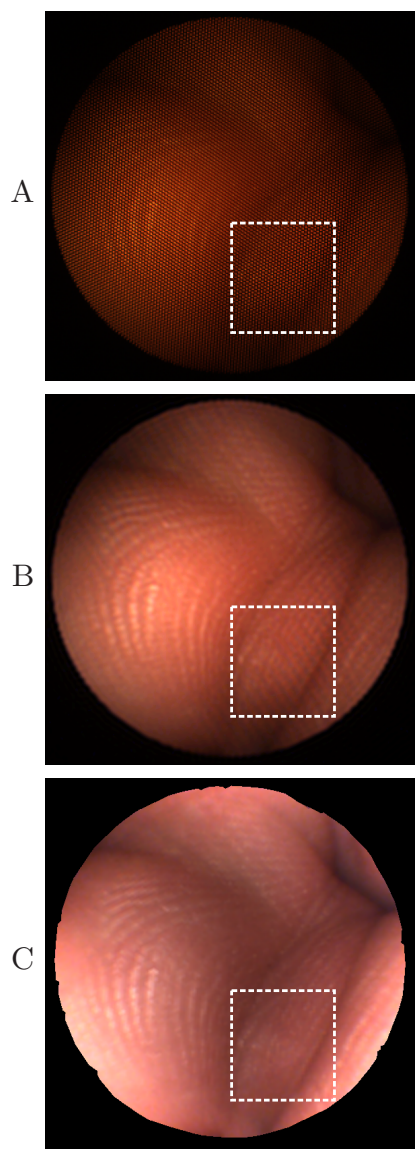

2

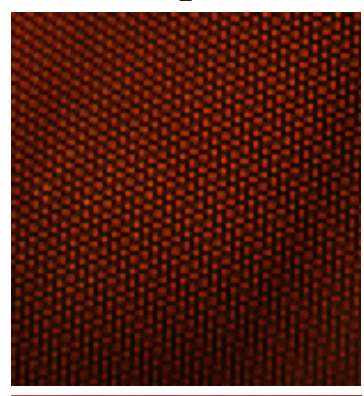

3
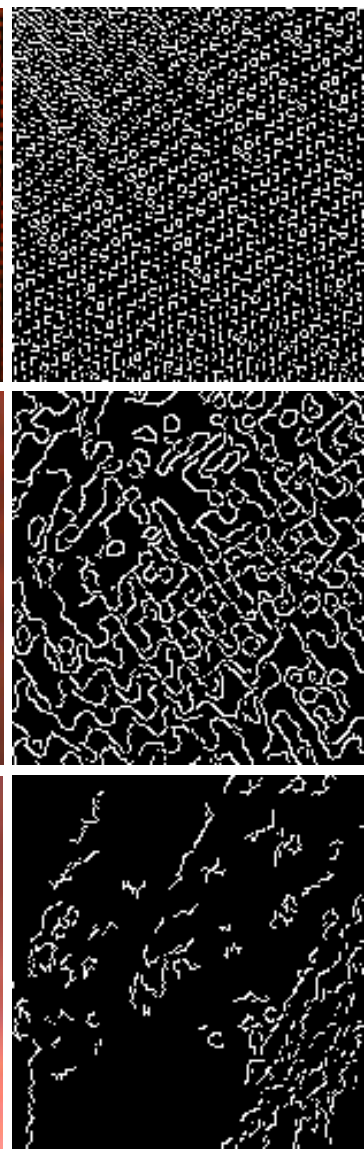

Fig. 2. Plain sensor data of fiberscopic snapshot of human skin (Part A1) with denoted enlarged section (Part A2) and result of Canny filter on red channel (Part A3). The homogeneous fine structure in Part A3 represents the gradient of the comb structure. The same data preprocessed by state of the art methods (row B). The partly crossing structure in the Canny result (B3) shows the overlaying effect of false colored strips from moiré. Row $\mathrm{C}$ gives an impression of the proposed approach without color irritation and with preserved relief texture of the hand illustrated by the Canny result in Part C3.

Due to the mounted fiberscope the visible part (aperture) is cropped to an extent of approx. $\oslash \approx 550$ px. The enlarged Part A2 shows that the image suffers from comb structure and strong mosaicking by the Bayer pattern. To depict the mixture of structures (hand relief, fiber comb and Bayer pattern), we use the Canny filter with constant lower (12) and upper (27) bound to post-process the red channel of the given sections. The mentioned effects in the plain data (fig. 2 , top row) of course lead to a homogeneous fine structure (fig. 2(A3)) mainly representing the comb structure. Image content can hardly be detected. 
Row $\mathrm{B}$ is the result of state of the art image processing, namely optimal fiberscopic low pass filtering, contrast enhancement, white balancing and tonal value correction. An automatic application of the latter two steps leads to false results because the processing already relies on wrong color distributions between the fibers' images. Although this work is not about color calibration and we do not care about some blue or red cast, we mention this effect here because it is responsible for the colored stripes overlaying the observed structure in the scene. Figure 2(B3) makes clear that in addition to the relief structure of the hand, several other components are mixed with the image.

The proposed method of physically motivated enhancement for color interpolation is applied in row $\mathrm{C}$. The particular difference to the state of the art can be seen in the enlarged parts fig. 2(C2) and fig. 2(C3). The grooves of the skin with an average distance of about $400 \mu \mathrm{m}$ can be separated clearly and appear without any colored texture fault. Manual adjustment of additional filters like in the previous processing is not necessary here. The color correction utilizes the knowledge of the position and intensity of each cell on the Bayer pattern and instead of inter-cell smoothing, the extended barycentric interpolation is performed to recover a pristine image.

The aperture's partly fringed border in the interpolation result (cf. fig. 2(C1)) results from the calibration step and can be reduced by smoothing the edge or completing the aperture's circle by adding some virtual centers.

\section{Conclusions and Future Work}

Structural precision and color information are of great importance for decisionmaking in endoscopy based procedures, such as CAD, incisions or navigation. Acquiring and transmitting image data via flexible devices with fiber bundles and digitizing it by a commonly used single-chip camera results in undesirable comb and false colored mosaicking. We introduced a new approach that takes into account the physical construction of the Bayer pattern on the digital sensor and calculates individual color factors for each spatially registered fiber area. This information supplements the extended barycentric interpolation. By processing the raw image data of the sensor it becomes possible to remove both the comb structure from the fiber bundle as well as the Bayer pattern mosaicking from the sensor in one single step while preserving important structures and textures in the scene. Optimizations like lookup tables enable real time processing of fiberscopic transmitted color images. The approach will improve the visual perception in small orifices for decision making and will add real value to existing hardware in medical environment.

Further improvement can be done in removing the effect of fringed borders in the interpolation result. We are currently working on the extension of a transmission model to consider the color coded mapping on the video sensor. This will help to quantify the improvement of our method. The color precision of the results will be approved by an independent concept of color calibration that could not be carried out yet. Also in-vivo performance remains to be assessed. 
In summary, the results of this work strongly suggests that an effective real time approach replacing several state of the art image processing steps is feasible to produce high quality colored fiberscopic images with digital sensors without false colors and moiré effects from mosaicking.

\section{Acknowledgement}

This work was supported by the Collaborative Research Center 603 (ModelBased Analysis and Visualization of Complex Scenes and Sensor Data) by the German Research Foundation (DFG).

\section{References}

1. Janssen, C.: Ein miniaturisiertes Endoskop-Stereomesssystem zur Strömungsvisual isierung in Kiesbetten. Master's thesis, Ruprecht-Karls-Univ. Heidelberg (2000)

2. Aslan, P., Kuo, R., Hazel, K., Babayan, R., Preminger, G.: Advances in digital imaging during endoscopic surgery. J. Endourology 13(4), 251-255 (1999)

3. Elter, M., Rupp, S., Winter, C.: Physically motivated reconstruction of fiberscopic images. In: IAPR/IEEE Intl. Conf. on Pattern Recognition, pp. 599-602. IEEE Computer Society Press, Los Alamitos (2006)

4. Lertrattanapanich, S., Bose, N.: High resolution image formation from low resolution frames using delaunay triangulation. IEEE Trans. Image Processing 11(12), $1427-$ 1441 (2002)

5. Winter, C., Elter, M., Rupp, S., Wittenberg, T.: Dynamic resolution enhancement for fiber optics. In: IASTED Intl. Conf. Signal and Image Processing, pp. 341-347 (2006)

6. Dickens, M.e.a.: Removal of optical fiber interference in color micro-endoscopic images. In: 11th IEEE Symp. Computer-Based Medical Systems, pp. 246-251. IEEE Computer Society Press, Los Alamitos (1998)

7. Winter, C., Rupp, S., Elter, M., Münzenmayer, C., Gerhäuser, H., Wittenberg, T.: Automatic adaptive enhancement for images obtained with fiberscopic endoscopes. IEEE Trans. Biomed. Eng. 53(10), 2035-2046 (2006)

8. Vakil, N., Knyrim, K., Everbach, E.: The appreciation of colour in endoscopy. Baillieres Clin. Gastroenterol. 1(5), 183 (1991) 